\title{
Front Matter: Volume 10847
}

, "Front Matter: Volume 10847," Proc. SPIE 10847, Optical Precision Manufacturing, Testing, and Applications, 1084701 (12 December 2018); doi: $10.1117 / 12.2521845$

SPIE Event: International Symposium on Optoelectronic Technology and SPIE. Application 2018, 2018, Beijing, China 


\title{
Optical Precision Manufacturing, Testing, and Applications
}

\author{
John W. McBride \\ JiuBin Tan \\ Sen Han \\ Xuejun Zhang \\ Editors
}

22-24 May 2018

Beijing, China

\section{Organized by}

Chinese Society for Optical Engineering (CSOE) (China)

Photoelectronic Technology Committee, Chinese Society of Astronautics (China)

Photoelectronic Industrialization Committee, CHIA (China)

Department of Cooperation and Coordination for Industry, Academe and Research, CHIA (China)

Science and Technology on Low-light-level Night Vision Laboratory (China)

Sponsored by

Division of Information and Electronic Engineering of Chinese Academy of Engineering (China) Chinese Society for Optical Engineering (CSOE) (China)

Published by

SPIE

\section{Volume 10847}


The papers in this volume were part of the technical conference cited on the cover and title page. Papers were selected and subject to review by the editors and conference program committee. Some conference presentations may not be available for publication. Additional papers and presentation recordings may be available online in the SPIE Digital Library at SPIEDigitalLibrary.org.

The papers reflect the work and thoughts of the authors and are published herein as submitted. The publisher is not responsible for the validity of the information or for any outcomes resulting from reliance thereon.

Please use the following format to cite material from these proceedings:

Author(s), "Title of Paper," in Optical Precision Manufacturing, Testing, and Applications, edited by John W. McBride, JiuBin Tan, Sen Han, Xuejun Zhang, Proceedings of SPIE Vol. 10847 (SPIE, Bellingham, WA, 2018) Seven-digit Article CID Number.

ISSN: 0277-786X

ISSN: 1996-756X (electronic)

ISBN: 9781510623361

ISBN: 9781510623378 (electronic)

Published by

SPIE

P.O. Box 10, Bellingham, Washington 98227-0010 USA

Telephone +1 3606763290 (Pacific Time) · Fax +1 3606471445

SPIE.org

Copyright (C) 2018, Society of Photo-Optical Instrumentation Engineers.

Copying of material in this book for internal or personal use, or for the internal or personal use of specific clients, beyond the fair use provisions granted by the U.S. Copyright Law is authorized by SPIE subject to payment of copying fees. The Transactional Reporting Service base fee for this volume is $\$ 18.00$ per article (or portion thereof), which should be paid directly to the Copyright Clearance Center (CCC), 222 Rosewood Drive, Danvers, MA 01923. Payment may also be made electronically through CCC Online at copyright.com. Other copying for republication, resale, advertising or promotion, or any form of systematic or multiple reproduction of any material in this book is prohibited except with permission in writing from the publisher. The CCC fee code is 0277$786 \mathrm{X} / 18 / \$ 18.00$.

Printed in the United States of America.

Publication of record for individual papers is online in the SPIE Digital Library.

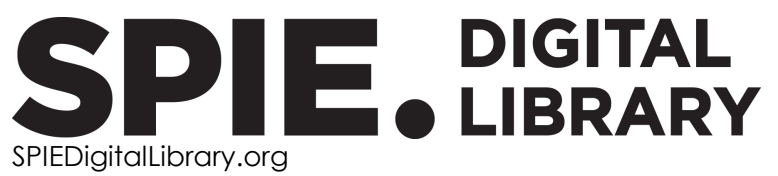

Paper Numbering: Proceedings of SPIE follow an e-First publication model. A unique citation identifier (CID) number is assigned to each article at the time of publication. Utilization of CIDs allows articles to be fully citable as soon as they are published online, and connects the same identifier to all online and print versions of the publication. SPIE uses a seven-digit CID article numbering system structured as follows:

- The first five digits correspond to the SPIE volume number.

- The last two digits indicate publication order within the volume using a Base 36 numbering system employing both numerals and letters. These two-number sets start with $00,01,02,03,04$, 05, 06, 07, 08, 09, 0A, OB ... 0Z, followed by 10-1Z, 20-2Z, etc. The CID Number appears on each page of the manuscript. 


\title{
Contents
}

\author{
vii Authors \\ ix Conference Committee \\ xi Introduction
}

OPTICAL PRECISION MANUFACTURING, TESTING, AND APPLICATIONS

1084702 The influence of the stray light on MTF in optical system [10847-1]

1084703 Method of optical vortex generation by cascaded spiral phase plates [10847-3]

1084704 Research on computer controlled ultra-precision polishing of freeform surfaces [10847-4]

1084705 Semiconductor laser thermal design optimization and analysis [10847-5]

1084706 High accuracy wavefront reconstruction with slope and coordinate compensation [10847-6]

1084707 Effect of precession mode on the surface error of optical components in bonnet polishing [10847-7]

1084708 Study on the design and preparation of wideband deep cutoff bandpass filter [10847-8]

1084709 A novel camera calibration method without distortion model [10847-9]

10847 OA Progress on fabrication and metrology technology study for M3M of TMT [10847-10]

10847 OB Laboratory results on the dependence of dark current upon environmental temperature variability for Satlantic's OCR504 radiometers [10847-11]

10847 OC Defects scattering imaging system of 20 inch PMTs' glass shell suitable for digital image processing [10847-12]

10847 OD Diffraction field simulation of waveguide grating coupler with variable periods [10847-13]

$10847 \mathrm{OE} \quad$ Influence of motion modes on surface quality in CCOS [10847-14]

10847 OF A measurement and separation method for perpendicular errors of rotary axes [10847-15]

10847 OG The application of adaptive theory in MEMS gyro error compensation [10847-16] 
$10847 \mathrm{OH} \quad$ Design and fabrication of high precision optical fiber coil based on temperature error model [10847-17]

10847 Ol Ratiometric wavelength monitor based on Mach-Zehnder interferometer over a 80nm wavelength range [10847-19]

10847 OJ Fiber coupling technology of white LED [10847-20]

10847 OK Optimization of large-aperture optics clean assembly method [10847-21]

$10847 \mathrm{OL} \quad$ Sand erosion durability of protective SiC thin film deposited by magnetron sputtering in the middle infrared bands [10847-22]

10847 OM Modeling for the thermal stress damage of the optical elements induced by high energy laser [10847-23]

10847 ON Pushing cavities to the edge for future gravitational wave detectors [10847-26]

$1084700 \quad$ Repeatability study on different interference systems [10847-27]

10847 OP Effect of magnetorheological processing parameters on polishing spots [10847-28]

$108470 Q \quad$ Optical design of LED street lamp based on freeform surface lens [10847-29]

10847 OR Research of controlling the optical face of CaF2 crystal elements on optical grinding and polishing [10847-30]

10847 OS Design of computer generated hologram for testing the wedged focus lens with large aperture [10847-31]

10847 OU Fabrication of subwavelength nanostructured grating for generating radially polarized light by using focused iron beam [10847-38]

10847 OV A wide range fiber turbidity sensor based on single photon detection technique with an optimal optical power [10847-40]

10847 OW Weighted fault-tolerant topology of wireless sensor networks [10847-41]

10847 OX Effects of fabrication errors on MTF and diffraction efficiency for binary optical lens [10847-42]

10847 OY Self-calibration and high-accuracy detection technology for the probability density of polarization state of a light field [10847-43]

$10847 \mathrm{OZ}$ The defocused helical structure of two superposed vortex beams [10847-44]

1084710 Development of an integrated freeform optics measurement system based on phase measuring deflectometry [10847-45] 
1084711 Optimization of transverse translation diverse phase retrieval for optical elements with mid-spatial frequency errors [10847-47]

1084712 High efficiency fabrication of 3D curved glass by full-motor-driving precision molding [10847-49]

1084713 Calibration of mercury lamp wavelength [10847-50] 
Proc. of SPIE Vol. 10847 1084701-6

Downloaded From: https://www.spiedigitallibrary.org/conference-proceedings-of-spie on 26 Apr 2023 Terms of Use: https://www.spiedigitallibrary.org/terms-of-use 


\section{Authors}

Numbers in the index correspond to the last two digits of the seven-digit citation identifier (CID) article numbering system used in Proceedings of SPIE. The first five digits reflect the volume number. Base 36 numbering is employed for the last two digits and indicates the order of articles within the volume. Numbers start with 00, 01, 02, 03, 04, 05, 06, 07, 08, 09, OA, OB...0Z, followed by 10-12, 20-2Z, etc.

Bai, Jian, 11

Bai, Jian-ming, 09

Bai, Longwen, OW

Bao, Zhengjun, OR

Bin, Boyi, 10

Brown, Daniel David, ON

Chen, Dan, 08

Chen, $\mathrm{HaO}, 06$

Chen, Jian, OM

Chen, Jiaojie, 06

Chen, Minsun, OM

Chen, Siyun, 06

Chen, Wenyuan, OG

Chen, Xian-hua, 07, OP

Chen, Yanjun, $\mathrm{OZ}$

Chen, Zihan, 12

Cheng, Xuemin, OX

Cheung, C. F., 04

Collins, Christopher, ON

Cui, Jian-Peng, OS

Dai, Xuebing, $\mathrm{OZ}$

Deng, Wen-hui, OP

Dovale-Alvarez, Miguel, ON

Du, Juan, 11

Du, Yifei, 12

Feng, Guojin, 13

Feng, Haihua, 06

Feng, Xianghua, OD

Freise, Andreas, ON

Gao, Wei, OE

Gui, Huaqiao, OV

Han, Kai, OM

Han, Sen, ON, $0 O$

$\mathrm{He}$, Guangzong, OL

$\mathrm{He}$, Jiahuan, 08

$\mathrm{He}$, Jianjun, $\mathrm{Ol}$

Ho, L. T., 04

Hou, Jing, OP

$\mathrm{Hu}$, Haifei, OA

$\mathrm{Hu}$, Haixiang, OA

Huang, Wei, 06

Huang, Xiao, 11

$\mathrm{Ji}$, Fang, $\mathrm{OE}$

Ji, Jianwei, OE

$\mathrm{Ji}$, Yiqin, 08

Jiang, Chenghui, 08

Jiang, Yugang, 08

Kong, Deyi, OV

Kong, Lingbao, OE, OF
Lagunas-Morales, José, OB

Lang, Tingting, $\mathrm{Ol}$

Li, B., 04

Li, Fang, $O Q$

$\mathrm{Li}$, Jianhui, OY, OZ

Li, Jie, 07, OP

Li, Shida, 08

Li, Xiao, OM

Li, Xinyi, 05

Li, Xiufei, 05

Li, Yanqiu, OY, OZ

Li, Yiyu, 06

Li, Zhigang, OR

Liang, Fengcheng, 13

Liang, Zhiqiang, 12

Liu, Bohan, $\mathrm{OH}$

Liu, Dandan, 08

Liu, Hao, OM

Liu, Huasong, 08

Liu, Jianguo, OV

Liu, Jie, OS

Liu, Jingqi, OC

Liu, Ke, OY

Liu, Lihui, OY, OZ

Liv, Sizhe, 05

Liu, Tong, 03

Liu, Zilong, 13

Long, Kai, OK

Lou, Zhaokai, OM

Lü, Liang, OV

LU, Min, OC

Lu, Zhongwen, OR

Luo, Xiao, OA

Luo, Yujie, 11

Luo, Yupeng, 11

LV, Song, 10

$\mathrm{Ma}$, Jun, $\mathrm{OH}$

Ma, Ping, OR, OS

$\mathrm{Ma}$, Yingjun, 02

Mei, Chao, 02

Mow-Lowry, Conor M., ON

Nan, Chunzhu, OG

Ning, Tianlei, OY

Pan, Wenhao, OY

Pei, Guoging, OK

Pu, Yunti, OR

Qi, Erhui, OA

Qian, Sen, OC

Qin, Feihu, OV 
Qu, Rui, 02

Quan, Wei, 05

Ren, Yiwen, 0J

Ren, Yuan, 03

Shao, Qiongling, 03

Song, Guangyi, Ol

Su, Shi, OJ

Sun, Gaofei, OJ

Tan, Qiaofeng, OX

Tang, Shouhong, 00

Tian, Weijian, OU

Wan, Wei, OV

Wan, Xinjun, 10

Wang, C. J., 04

Wang, Chen, 03

Wang, Haoyu, ON

Wang, Hong-xiang, OP

Wang, Hualin, OC

Wang, Huanqin, OV

Wang, Hui, OK

Wang, Jiqiang, OJ

Wang, Junhua, OE

Wang, Liping, OE

Wang, Lishuan, 08

Wang, Mengyao, ON

Wang, Shuo, OW

Wang, Shuxin, Ol

Wang, Xibin, 12

Wang, Ye, $O Q$

Wang, Ye, $\mathrm{OZ}$

Wang, Yiwen, OJ

Wang, Yueze, $\mathrm{OH}$

Wang, Zhi le, OC

Wei, Zhengtong, OD

Wen, Zhong-jiang, 07

Wu, Houping, 13

Wu, Shaogiang, OD

$\mathrm{Wu}$, Xiaole, $\mathrm{OH}$

Xie, Shuping, 10

Xing, Xiaogang, $O B$

$X \cup, H \cup a, O S$

$X U, M i n, O E, O F$

$X U, P ., 04$

Xue, Jun, OL

Yan, Aqi, 02

Yan, Baozhu, OM

Yan, Ding-Yao, OS

Yang, Yi, OM

Yi, Congzhi, OK

Yu, Fajun, OV

Zhai, Yueyang, 05

Zhang, Bo, 00

Zhang, Guoyu, OJ

Zhang, Haitao, $\mathrm{OE}$

Zhang, Jian, OJ

Zhang, Linghua, 00

Zhang, Ning, OS

Zhang, Qiaoxiang, 13

Zhang, Tianxing, OL

Zhang, Zheng, OK
Zhao, Feng, OC

Zhao, Hui, Ol

Zhao, Lei, 11

Zhao, Yu-hui, 09

Zheng, Chundi, 13

Zheng, Meng, OY

Zheng, Tieqiao, OX

Zhong, Bo, 07, OP

Zhou, Guodong, $\mathrm{OZ}$

Zhou, Guozun, OU

Zhou, Jia, 12

Zhou, Junhui, OG

Zhou, Panyu, OF

Zhou, Qing, OF

Zhou, Tianfeng, 12

Zhou, Xiangdong, 11

Zhu, Heng, OR

Zhu, Yao, OC

Zhuang, Chengjin, 00

Zhuang, Songlin, 10

viii

Proc. of SPIE Vol. 10847 1084701-8 


\title{
Conference Committee
}

\author{
Conference Chair
}

Guangjun Zhang, Southeast University (China)

\section{Conference Co-chairs}

Junhao Chu, Shanghai Institute of Technical Physics (China)

Qionghai Dai, Tsinghua University (China)

Dianyuan Fan, Shenzhen University (China)

Jiancheng Fang, Beihang University (China)

Gu Min, Royal Melbourne Institute of Technology University (Australia)

Desheng Jiang, Wuhan University of Technology (China)

Huilin Jiang, Changchun University of Science and Technology (China)

Lin Li, The University of Manchester (United Kingdom)

Yueguang Lv, Chinese Academy of Engineering (China)

Zhejin Liu, National University of Defense Technology (China)

Wang Xiaomo, China Academy of Electronics and Information Technology (China)

Huaming Wang, Beihang University (China)

Lijun Wang, Changchun Institute of Optics, Fine Mechanics and Physics (China)

Wei Wang, China Aerospace Science and Technology Corporation (China)

Jianyu Wang, Shanghai Branch of Chinese Academy of Sciences (China)

Zuyan Xu, The Technical Institute of Physics and Chemistry (China)

Jiubin Tan, Harbin Institute of Technology (China)

Jianquan Yao, Tianjin University (China)

Hao Yin, China Electronic Systems Engineering Corporation (China)

Shaohua Yu, Wuhan Research Institute of Posts and Telecommunications (China)

Renhe Zhang, Institute of Acoustics (China)

Zisen Zhao, Wuhan Research Institute of Posts and Telecommunications (China)

Liwei Zhou, Beijing Institute of Technology (China)

Shouhuan Zhou, North China Research Institute of Electro-optics (China)

Zhongliang Zhu, Southwest Electronic Telecom Technology Research Institute (China) 
Program Committee

Byoungho Lee, Seoul National University (Korea, Republic of)

Liangcai Cao, Tsinghua University (China)

Weibiao Chen, Shanghai Institute of Optics and Fine Mechanics

(China)

Haimei Gong, Shanghai Institute of Technical Physics (China)

Sen Han, University of Shanghai for Science and Technology (China)

Huikai Xie, University of Florida (United States)

John McBride, University of Southampton (United Kingdom)

Yanbiao Liao, Tsinghua University (China)

Dong Liu, Zhejiang University (China)

Jian Liu, Harbin Institute of Technology (China)

Jin Lu, Tianjin Jinhang Institute of Technical Physics (China)

Mircea Guina, Tampere University of Technology (Finland)

Shibin Jiang, AdValue Photonics, Inc. (United States)

Guohai Situ, Shanghai Institute of Optics and Fine Mechanics (China)

Hongbo Sun, Tsinghua University (China)

Yongtian Wang, Beijing Institute of Technology (China)

Yuelin Wang, Shanghai Institute of Microsystem and Information

Technology (China)

Renhe Zhang, Institute of Acoustics (China)

Xuejun Zhang, Changchun Institute of Optics, Fine Mechanics and

Physics (China)

Pu Zhou, National University of Defense Technology (China)

Zhongliang Zhu, Southwest Electronic Telecom Technology Research Institute (China)

\section{Session Chairs}

1 Optical Precision Manufacturing, Technology, and Applications I Sen Han, University of Shanghai for Science and Technology (China)

2 Optical Precision Manufacturing, Technology, and Applications II John W. McBride, University of Southampton (United Kingdom)

3 Optical Precision Manufacturing, Technology, and Applications III W. B. Lee, The Hong Kong Polytechnic University (Hong Kong, China)

4 Optical Precision Manufacturing, Technology, and Applications IV Jian Liu, Harbin Institute of Technology (China)

5 Optical Precision Manufacturing, Technology, and Applications $V$ Lingbao Kong, Fudan University (China) 


\section{Introduction}

The International Symposium on Optoelectronic Technology and Application 2018 (OTA 2018) is the annual conference of the Chinese Society for Optical Engineering. It continues to be one of the largest academic and industrial conferences in the field of optical and optoelectronic technology in China. This year's program included academic exchanges, industry exhibitions, and cooperation negotiations together in one event. There were five technical conferences, seven exhibition themes, and 600 technical negotiations. We sincerely hope that this event continues to promote research and development of optoelectronic technology and to enhance international cooperation in the optical and optoelectronic fields.

OTA 2018 was sponsored by The Division of Information and Electronic Engineering of the Chinese Academy of Engineering (China), and The Chinese Society for Optical Engineering (CSOE) (China). The conference was organized by the Chinese Society for Optical Engineering (CSOE) (China), the Photoelectronic Technology Committee, the Chinese Society of Astronautics (China), the Photoelectronic Industrialization Committee, CHIA (China), the Department of Cooperation and Coordination for Industry, Academe, and Research, CHIA (China), and the Science and Technology on Low-light-level Night Vision Laboratory (China). We received more than 759 contributions from more than 15 countries, including the United States, United Kingdom, Germany, France, Spain, Australia, Canada, Mexico, Brazil, Japan, Republic of Korea, Thailand, Singapore, Russian Federation and China. There were more than 400 contributions published in SPIE Proceedings, including 70 contributions from invited speakers. After careful discussion, six keynote speeches were selected and presented by famous scientists from the United States, United Kingdom, Republic of Korea, and China. There were 138 excellent invited talks, 45 from overseas, that reflected first-class level in the field of optics and photonics technology. On behalf of the OTA 2018 Organizing Committee, I would like to express thanks to all the invited speakers and authors for their contributions and support.

Finally, on behalf of the other Co-chairmen and the Organizing Committee, I would like to heartily thank our sponsors and cooperating organizers for all they have done for the conference, and to all of the participants and friends for their interests and efforts in helping us to make the conference a success. Thanks also to the Program Committee for their effective work and valuable advice, especially the Secretariat, and to the staff of SPIE for their tireless efforts and outstanding service preparing and publishing the proceedings.

We hope to see you next year!

\section{Guangjun Zhang}


Proc. of SPIE Vol. 10847 1084701-12 Downloaded From: https://www.spiedigitallibrary.org/conference-proceedings-of-spie on 26 Apr 2023
Terms of Use: https://www.spiedigitallibrary.org/terms-of-use 